TRANSACTIONS OF THE

AMERICAN MATHEMATICAL SOCIETY

Volume 361, Number 4, April 2009, Pages 1949-1961

S 0002-9947(08)04648-5

Article electronically published on October 22, 2008

\title{
ON THE NONEXISTENCE OF NONTRIVIAL INVOLUTIVE $n$-HOMOMORPHISMS OF $C^{\star}$-ALGEBRAS
}

\author{
EFTON PARK AND JODY TROUT
}

\begin{abstract}
An $n$-homomorphism between algebras is a linear map $\phi: A \rightarrow B$ such that $\phi\left(a_{1} \cdots a_{n}\right)=\phi\left(a_{1}\right) \cdots \phi\left(a_{n}\right)$ for all elements $a_{1}, \ldots, a_{n} \in A$. Every homomorphism is an $n$-homomorphism for all $n \geq 2$, but the converse is false, in general. Hejazian et al. (2005) ask: Is every $*$-preserving $n$-homomorphism between $C^{\star}$-algebras continuous? We answer their question in the affirmative, but the even and odd $n$ arguments are surprisingly disjoint. We then use these results to prove stronger ones: If $n>2$ is even, then $\phi$ is just an ordinary $*$-homomorphism. If $n \geq 3$ is odd, then $\phi$ is a difference of two orthogonal *-homomorphisms. Thus, there are no nontrivial $*$-linear $n$-homomorphisms between $C^{\star}$-algebras.
\end{abstract}

\section{INTRODUCTION}

Let $A$ and $B$ be algebras and $n \geq 2$ an integer. A linear map $\phi: A \rightarrow B$ is an $n$-homomorphism if for all $a_{1}, a_{2}, \ldots, a_{n} \in A$,

$$
\phi\left(a_{1} a_{2} \cdots a_{n}\right)=\phi\left(a_{1}\right) \phi\left(a_{2}\right) \cdots \phi\left(a_{n}\right) .
$$

A 2-homomorphism is then just a homomorphism, in the usual sense, between algebras. Furthermore, every homomorphism is clearly also an $n$-homomorphism for all $n \geq 2$, but the converse is false, in general. The concept of $n$-homomorphism was studied for complex algebras by Hejazian, Mirzavaziri, and Moslehian [7. This concept also makes sense for rings and (semi)groups. For example, an $A E_{n}$-ring is a ring $R$ such that every additive endomorphism $\phi: R \rightarrow R$ is an $n$-homomorphism; Feigelstock [4, 5] classified all unital $A E_{n}$-rings.

In [7, Hejazian et al. ask: Is every $*$-preserving $n$-homomorphism between $C^{\star}$ algebras continuous? We answer in the affirmative by proving that every involutive $n$-homomorphism $\phi: A \rightarrow B$ between $C^{\star}$-algebras is in fact norm contractive: $\|\phi\| \leq 1$. Surprisingly, the arguments for the even and odd $n$ cases are disjoint and, thus, are discussed in different sections. When $n=3$, automatic continuity is reported by Bračič and Moslehian [2, but note that the proof of their Theorem 2.1 does not extend to the nonunital case since the unitization of a 3-homomorphism is not a 3 -homomorphism, in general.

Received by the editors April 6, 2007.

2000 Mathematics Subject Classification. Primary 46L05; Secondary 47B99, 47L30.

(C)2008 American Mathematical Society

Reverts to public domain 28 years from publication 
Using these automatic continuity results, we prove the following stronger results: If $n>2$ is even, every $*$-linear $n$-homomorphism $\phi: A \rightarrow B$ between $C^{\star}$-algebras is in fact a $*$-homomorphism. If $n \geq 3$ is odd, every $*$-linear $n$ homomorphism $\phi: A \rightarrow B$ is a difference $\phi(a)=\psi_{1}(a)-\psi_{2}(a)$ of two orthogonal $*$-homomorphisms $\psi_{1} \perp \psi_{2}$. Regardless, for all integers $n \geq 3$, every positive linear $n$-homomorphism is a $*$-homomorphism. Note that if $\psi$ is a $*$-homomorphism, then $-\psi=0-\psi$ is a norm contractive $*$-preserving 3 -homomorphism that is not positive linear.

There is also a dichotomy between the unital and nonunital cases. When the domain algebra $A$ is unital, there is a simple representation of an $n$ homomorphism as a certain $n$-potent multiple of a homomorphism (discussed in the Appendix). The nonunital case is more subtle. For example, if $A$ and $B$ are nonunital (Banach) algebras such that $A^{n}=B^{n}=\{0\}$, then every linear map $L: A \rightarrow B$ (bounded or unbounded) is, trivially, an $n$-homomorphism (see Examples 2.5 and 4.3 of [7]).

The outline of the paper is as follows: In Section 2, we prove automatic continuity for the even case and in Section 3 for the odd case. In Section 4, we prove our nonexistence results. A key fact in many of our proofs is the Cohen Factorization Theorem [3] of $C^{\star}$-algebras. (See Proposition 2.33 in 8 for an elementary proof of this important result.) Finally, in Appendix A, we collect some facts about $n$-potents that we need.

The authors would like to thank Dana Williams and Tom Shemanske for their helpful comments and suggestions.

\section{Automatic continuity: The even case}

In this section, we prove that when $n>2$ is even, every involutive (i.e., *-linear) $n$-homomorphism between $C^{\star}$-algebras is completely positive and norm contractive, which generalizes the well-known result for $*$-homomorphisms $(n=2)$. Recall that a linear map $\theta: A \rightarrow B$ between $C^{\star}$-algebras is positive if $a \geq 0$ implies $\theta(a) \geq 0$ or, equivalently, for every $a \in A$ there is a $b \in B$ such that $\theta\left(a^{*} a\right)=b^{*} b$. We say that $\theta$ is completely positive if, for all $k \geq 1$, the induced map $\theta_{k}: M_{k}(A) \rightarrow M_{k}(B)$, $\theta_{k}\left(\left(a_{i j}\right)\right)=\left(\theta\left(a_{i j}\right)\right)$, on $k \times k$ matrices is positive.

Theorem 2.1. Let $\mathcal{H}$ be a Hilbert space. If $n \geq 2$ is even, then every involutive n-homomorphism from a $C^{\star}$-algebra $A$ into $\mathcal{B}(\mathcal{H})$ is completely positive.

Proof. Let $\phi: A \rightarrow \mathcal{B}(\mathcal{H})$ be an involutive $n$-homomorphism. We may assume $n=2 k>2$. Let $\langle\cdot, \cdot\rangle$ denote the inner product on $\mathcal{H}$. By Stinespring's Theorem 9 ] (see Prop. II.6.6 in [1]), $\phi$ is completely positive if and only if for any $m>1$ and elements $a_{1}, \ldots, a_{m} \in A$ and vectors $v_{1}, \ldots, v_{m} \in \mathcal{H}$ we have

$$
\sum_{i, j=1}^{m}\left\langle\phi\left(a_{i}^{*} a_{j}\right) v_{j}, v_{i}\right\rangle \geq 0 .
$$

We proceed as follows: For each $1 \leq i \leq m$ use the Cohen Factorization Theorem [3] to factor $a_{i}=a_{i 1} \cdots a_{i k}$ into a product of $k$ elements. Thus, their adjoints factor 
as $a_{i}^{*}=a_{i k}^{*} \cdots a_{i 1}^{*}$. Since $n=2 k$, we compute

$$
\begin{aligned}
\sum_{i, j=1}^{m}\left\langle\phi\left(a_{i}^{*} a_{j}\right) v_{j}, v_{i}\right\rangle & =\sum_{i, j=1}^{m}\left\langle\phi\left(a_{i k}^{*} \cdots a_{i 1}^{*} a_{j 1} \cdots a_{j k}\right) v_{j}, v_{i}\right\rangle \\
& =\sum_{i, j=1}^{m}\left\langle\phi\left(a_{i k}\right)^{*} \cdots \phi\left(a_{i 1}\right)^{*} \phi\left(a_{j 1}\right) \cdots \phi\left(a_{j k}\right) v_{j}, v_{i}\right\rangle \\
& =\left\langle\sum_{j=1}^{m} \phi\left(a_{j 1}\right) \cdots \phi\left(a_{j k}\right) v_{j}, \sum_{i=1}^{m} \phi\left(a_{i 1}\right) \cdots \phi\left(a_{i k}\right) v_{j}\right\rangle \\
& =\langle x, x\rangle \geq 0,
\end{aligned}
$$

where $x=\sum_{i=1}^{m} \phi\left(a_{i 1}\right) \cdots \phi\left(a_{i k}\right) v_{i} \in \mathcal{H}$. The result now follows.

Even though the previous result is a corollary of the more general theorem below, we have included it because the proof technique is different.

Lemma 2.2. Let $\phi: A \rightarrow B$ be an n-homomorphism. Then, for all $k \geq 1$, the induced maps $\phi_{k}: M_{k}(A) \rightarrow M_{k}(B)$ on $k \times k$ matrices are $n$-homomorphisms. Moreover, if $\phi$ is involutive $\left(\phi\left(a^{*}\right)=\phi(a)^{*}\right)$, then each $\phi_{k}$ is also involutive.

Proof. Given $n$ matrices $a^{1}=\left(a_{i j}^{1}\right), \ldots, a^{n}=\left(a_{i j}^{n}\right)$ in $M_{k}(A)$, we can express their product $a^{1} a^{2} \cdots a^{n}=\left(a_{i j}\right)$, where the $(i, j)$-th entry $a_{i j}$ is given by the formula

$$
a_{i j}=\sum_{m_{1}, \cdots, m_{n-1}=1}^{k} a_{i m_{1}}^{1} a_{m_{1} m_{2}}^{2} \cdots a_{m_{n-1} j}^{n} .
$$

Since $\phi_{k}\left(a^{1} a^{2} \cdots a^{n}\right)=\left(\phi\left(a_{i j}\right)\right)$ by definition and

$$
\begin{aligned}
\phi\left(a_{i j}\right) & =\sum_{m_{1}, \cdots, m_{n-1}=1}^{k} \phi\left(a_{i m_{1}}^{1} a_{m_{1} m_{2}}^{2} \cdots a_{m_{n-1} j}^{n}\right) \\
& =\sum_{m_{1}, \cdots, m_{n-1}=1}^{k} \phi\left(a_{i m_{1}}^{1}\right) \phi\left(a_{m_{1} m_{2}}^{2}\right) \cdots \phi\left(a_{m_{n-1} j}^{n}\right) \\
& =\left[\phi_{k}\left(a^{1}\right) \phi_{k}\left(a^{2}\right) \cdots \phi_{k}\left(a^{n}\right)\right]_{i j}
\end{aligned}
$$

it follows that $\phi_{k}: M_{k}(A) \rightarrow M_{k}(B)$ is an $n$-homomorphism. Now suppose that $\phi$ is involutive. We compute for all $a=\left(a_{i j}\right) \in M_{k}(A)$ :

$$
\phi_{k}\left(a^{*}\right)=\phi_{k}\left(\left(a_{j i}^{*}\right)\right)=\left(\phi\left(a_{j i}^{*}\right)\right)=\left(\phi\left(a_{j i}\right)^{*}\right)=\phi_{k}(a)^{*}
$$

and hence each $\phi_{k}: M_{k}(A) \rightarrow M_{k}(B)$ is involutive.

Theorem 2.3. Let $\phi: A \rightarrow B$ be an involutive $n$-homomorphism between $C^{\star}$ algebras. If $n \geq 2$ is even, then $\phi$ is completely positive. Thus, $\phi$ is bounded.

Proof. We may assume $n=2 k>2$. Since $\phi$ is linear, we want to show that for every $a \in A$ we have $\phi\left(a^{*} a\right) \geq 0$. By the Cohen Factorization Theorem, for any $a \in A$ we can find $a_{1}, \ldots, a_{k} \in A$ such that the factorization $a=a_{1} \cdots a_{k}$ holds. Thus, the adjoint factors as $a^{*}=a_{k}^{*} \cdots a_{1}^{*}$. Since $n=2 k$ and $\phi$ is $n$-multiplicative 
and $*$-preserving,

$$
\begin{aligned}
\phi\left(a^{*} a\right) & =\phi\left(a_{k}^{*} \cdots a_{1}^{*} a_{1} \cdots a_{k}\right) \\
& =\phi\left(a_{k}\right)^{*} \cdots \phi\left(a_{1}\right)^{*} \phi\left(a_{1}\right) \cdots \phi\left(a_{k}\right) \\
& =\left(\phi\left(a_{1}\right) \cdots \phi\left(a_{k}\right)\right)^{*}\left(\phi\left(a_{1}\right) \cdots \phi\left(a_{k}\right)\right) \\
& =b^{*} b \geq 0,
\end{aligned}
$$

where $b=\phi\left(a_{1}\right) \cdots \phi\left(a_{k}\right) \in B$. Thus, $\phi$ is a positive linear map. By the previous lemma, all of the induced maps $\phi_{k}: M_{k}(A) \rightarrow M_{k}(B)$ on $k \times k$ matrices are involutive $n$-homomorphisms and are positive. Hence, $\phi$ is completely positive and therefore bounded [1].

We now wish to show that if $n \geq 2$ is even, then an involutive $n$-homomorphism is actually norm-contractive. First, we will need generalizations of the familiar $C^{\star}$-identity appropriate for $n$-homomorphisms.

Lemma 2.4. Let $A$ be a $C^{\star}$-algebra. For all $k \geq 1$, we have that

$$
\left\{\begin{array}{l}
\|x\|^{2 k}=\left\|\left(x^{*} x\right)^{k}\right\|, \\
\|x\|^{2 k+1}=\left\|x\left(x^{*} x\right)^{k}\right\|
\end{array}\right.
$$

for all $x \in A$.

Proof. In the even case, we easily have that

$$
\|x\|^{2 k}=\left(\|x\|^{2}\right)^{k}=\left\|x^{*} x\right\|^{k}=\left\|\left(x^{*} x\right)^{k}\right\|
$$

by the functional calculus since $x^{*} x \geq 0$. In the odd case, we compute again using the $C^{\star}$-identity and functional calculus:

$$
\begin{aligned}
\left\|x\left(x^{*} x\right)^{k}\right\|^{2} & =\left\|\left(x\left(x^{*} x\right)^{k}\right)^{*}\left(x\left(x^{*} x\right)^{k}\right)\right\| \\
& =\left\|\left(x^{*} x\right)^{k} x^{*} x\left(x^{*} x\right)^{k}\right\| \\
& =\left\|\left(x^{*} x\right)^{2 k+1}\right\|=\left\|\left(x^{*} x\right)\right\|^{2 k+1} \\
& =\left(\|x\|^{2}\right)^{2 k+1}=\left(\|x\|^{2 k+1}\right)^{2}
\end{aligned}
$$

the result follows by taking square roots.

Theorem 2.5. Let $\phi: A \rightarrow B$ be an involutive $n$-homomorphism of $C^{\star}$-algebras. If $\phi$ is bounded, then $\phi$ is norm-contractive $(\|\phi\| \leq 1)$.

Proof. Suppose $n=2 k$ is even. Then for all $x \in A$ we have

$$
\phi\left(\left(x^{*} x\right)^{k}\right)=\phi\left(x^{*} x \cdots x^{*} x\right)=\left(\phi\left(x^{*}\right) \phi(x)\right)^{k}=\left(\phi(x)^{*} \phi(x)\right)^{k} .
$$

Thus by the previous lemma,

$$
\begin{aligned}
\|\phi(x)\|^{n} & =\|\phi(x)\|^{2 k} \\
& =\left\|\left(\phi(x)^{*} \phi(x)\right)^{k}\right\|=\left\|\phi\left(\left(x^{*} x\right)^{k}\right)\right\| \\
& \leq\|\phi\|\left\|\left(x^{*} x\right)^{k}\right\|=\|\phi\|\|x\|^{2 k}=\|\phi\|\|x\|^{n},
\end{aligned}
$$

which implies that $\|\phi\| \leq 1$ by taking $n$-th roots.

The proof for the odd case $n=2 k+1$ is similar. 


\section{Automatic continuity: The odd Case}

The positivity methods above do not work when $n$ is odd, since the negation of a $*$-homomorphism defines an involutive 3 -homomorphism that is (completely) bounded, but not positive. We need the following slight generalization of Lemma 3.5 of Harris $[\underline{6}$.

Lemma 3.1. Let $A$ be $a C^{\star}$-algebra and let $\lambda \neq 0$ and $k \geq 1$. If $a \in A$, then $\lambda \in \sigma\left(\left(a^{*} a\right)^{k}\right)$ if and only if there does not exist an element $c \in A$ with

$$
c\left(\lambda-\left(a^{*} a\right)^{k}\right)=a .
$$

Proof. If $\lambda \notin \sigma\left(\left(a^{*} a\right)^{k}\right)$, then $c=a\left(\lambda-\left(a^{*} a\right)^{k}\right)^{-1} \in A$ satisfies

$$
c\left(\lambda-\left(a^{*} a\right)^{k}\right)=a\left(\lambda-\left(a^{*} a\right)^{k}\right)^{-1}\left(\lambda-\left(a^{*} a\right)^{k}\right)=a,
$$

and so (11) holds.

On the other hand, if $\lambda \in \sigma\left(\left(a^{*} a\right)^{k}\right)$ then, by the commutative functional calculus, there is a sequence $\left\{b_{m}\right\}_{1}^{\infty}$ in the unitization $A^{+}$with $b_{m} \nrightarrow 0$ but $d_{m}={ }_{\text {def }}\left(\lambda-\left(a^{*} a\right)^{k}\right) b_{m} \rightarrow 0$. Since $\lambda \neq 0$ we must have

$$
a^{*}\left(a a^{*}\right)^{k-1}\left(a b_{m}\right)=\left(a^{*} a\right)^{k} b_{m}=\lambda b_{m}-d_{m} \nrightarrow 0,
$$

which implies $a b_{m} \nrightarrow 0$. Hence, there does not exist an element $c \in A$ that can satisfy equation (11), since this would imply that

$$
a b_{m}=c\left(\lambda-\left(a^{*} a\right)^{k}\right) b_{m} \rightarrow 0,
$$

which is a contradiction. This proves the lemma.

We now prove automatic continuity for involutive $n$-homomorphisms of $C^{\star}$ algebras for all odd values of $n$. Note that we do not assume that $A$ is unital, nor do we appeal to the unitization $\phi^{+}: A^{+} \rightarrow B^{+}$of $\phi$, which is not an $n$ homomorphism, in general.

Theorem 3.2. Let $\phi: A \rightarrow B$ be an involutive $n$-homomorphism between $C^{\star}$ algebras. If $n \geq 3$ is odd, then $\|\phi\| \leq 1$, i.e., $\phi$ is norm-contractive.

Proof. Let $n=2 k+1$ where $k \geq 1$. Given any $a \in A$ and $\lambda>0$ such that $\lambda \notin \sigma\left(\left(a^{*} a\right)^{k}\right)$, there is, by the previous lemma, an element $c \in A$ such that

$$
a=c\left(\lambda-\left(a^{*} a\right)^{k}\right)=\left(\lambda c-c\left(a^{*} a\right)^{k}\right) .
$$

Noting that $c\left(a^{*} a\right)^{k}$ is a product of $2 k+1=n$ elements in $A$, and $\phi$ is a $*$-linear $n$-homomorphism, we compute:

$$
\begin{aligned}
\phi(a) & =\phi\left(\lambda c-c\left(a^{*} a\right)^{k}\right)=\lambda \phi(c)-\phi\left(c\left(a^{*} a\right)^{k}\right) \\
& =\lambda \phi(c)-\phi(c)\left(\phi(a)^{*} \phi(a)\right)^{k}=\phi(c)\left(\lambda-\left(\phi(a)^{*} \phi(a)\right)^{k}\right),
\end{aligned}
$$

which yields that there is an element $\phi(c) \in B$ with:

$$
\phi(c)\left(\lambda-\left(\phi(a)^{*} \phi(a)\right)^{k}\right)=\phi(a) .
$$

By the previous lemma, we conclude that $\lambda \notin \sigma\left(\left(\phi(a)^{*} \phi(a)\right)^{k}\right)$. Thus, we have shown the following inclusion of spectra:

$$
\sigma\left(\left(\phi(a)^{*} \phi(a)\right)^{k}\right) \subseteq \sigma\left(\left(a^{*} a\right)^{k}\right) \cup\{0\} .
$$


Therefore, by the spectral radius formula [1, II.1.6.3] and the generalization of the $C^{\star}$-identity in Lemma 2.4, we must deduce that:

$$
\begin{aligned}
\|\phi(a)\|^{2 k} & =\left\|\left(\phi(a)^{*} \phi(a)\right)^{k}\right\| \\
& =r\left(\left(\phi(a)^{*} \phi(a)\right)^{k}\right) \leq r\left(\left(a^{*} a\right)^{k}\right) \\
& =\left\|\left(a^{*} a\right)^{k}\right\|=\|a\|^{2 k},
\end{aligned}
$$

which implies that $\|\phi(a)\| \leq\|a\|$ for all $a \in A$, as desired.

Note that the argument in the previous proof does not work for $n=2 k$ even, since we would need to employ $\left(a^{*} a\right)^{k-1} a$ which is a product of $2 k-1=n-1$ elements as needed, but not self-adjoint, in general. Thus, we could not appeal to the spectral radius formula for self-adjoint elements and Lemma 3.1 would not apply. Hence, the even and odd $n$ arguments are essentially disjoint.

\section{NONEXISTENCE OF NONTRIVAL INVOLUTIVE $n$-HOMOMORPHISMS OF $C^{\star}$-ALGEBRAS}

Our first main result is the nonexistence of nontrivial $n$-homomorphisms on unital $C^{\star}$-algebras for all $n \geq 3$. We do the unital case first since it is much simpler to prove and helps to frame the argument for the nonunital case.

Theorem 4.1. Let $\phi: A \rightarrow B$ be an involutive $n$-homomorphism between the $C^{\star}$ algebras $A$ and $B$, where $A$ is unital. If $n \geq 2$ is even, then $\phi$ is $a *$-homomorphism. If $n \geq 3$ is odd, then $\phi$ is the difference $\phi(a)=\psi_{1}(a)-\psi_{2}(a)$ of two orthogonal *-homomorphisms $\psi_{1} \perp \psi_{2}: A \rightarrow B$.

Proof. In either case, by Proposition A.1, the element $e=\phi(1) \in B$ is an $n$-potent $\left(e^{n}=e\right)$ and is self-adjoint because

$$
e=\phi(1)=\phi\left(1^{*}\right)=\phi(1)^{*}=e^{*} .
$$

Also, there is an associated algebra homomorphism $\psi: A \rightarrow B$ defined for all $a \in A$ by the formula

$$
\psi(a)=e^{n-2} \phi(a)=\phi(a) e^{n-2}
$$

such that $\phi(a)=e \psi(a)=\psi(a) e$. In either case, $\psi$ is $*$-linear since $\phi$ is $*$-linear and $e$ is self-adjoint and commutes with the range of $\phi$ :

$$
\psi\left(a^{*}\right)=e^{n-2} \phi\left(a^{*}\right)=e^{n-2} \phi(a)^{*}=\left(e^{n-2} \phi(a)\right)^{*}=\psi(a)^{*} .
$$

Now, if $n=2 k$ is even, $e=e^{n}=\left(e^{k}\right)^{*} e^{k} \geq 0$ and so $e=p$ is a projection. Thus, $\phi(a)=p \psi(a)=\psi(a) p=p \psi(a) p$ is a $*$-homomorphism. If $n \geq 3$ is odd, then by Lemma A.8 $e$ is the difference of two orthogonal projections $e=p_{1}-p_{2}$ which must commute with both $\psi$ and $\phi$ by the functional calculus. Define $\psi_{1}, \psi_{2}: A \rightarrow B$ by $\psi_{i}(a)=p_{i} \psi(a) p_{i}$ for all $a \in A$ and $i=1,2$. Then $\psi_{2} \perp \psi_{2}$ are orthogonal *-homomorphisms, and

$$
\psi_{1}(a)-\psi_{2}(a)=p_{1} \psi(a)-p_{2} \psi(a)=e \psi(a)=\phi(a)
$$

for all $a \in A$, from which the desired result follows. 
Corollary 4.2. Let $\phi: A \rightarrow B$ be a linear map between $C^{\star}$-algebras. If $A$ is unital, the following are equivalent for all integers $n \geq 2$ :

(a) $\phi$ is a $*$-homomorphism.

(b) $\phi$ is a positive $n$-homomorphism.

(c) $\phi$ is an involutive $n$-homomorphism and $\phi(1) \geq 0$.

Proof. Clearly (a) $\Longrightarrow$ (b) $\Longrightarrow$ (c). If $n \geq 2$ is even, then (c) $\Longrightarrow$ (a) by the previous result. If $n \geq 3$ is odd, then by the previous result, we only need to show that $\phi$ is positive. Let $n=2 k+1$. Given any $a \in A$, by the Cohen Factorization Theorem, we can write $a=a_{1} \cdots a_{k}$. Since $\phi(1) \geq 0$, by hypothesis, and $n=2 k+1$, we compute:

$$
\begin{aligned}
\phi\left(a^{*} a\right) & =\phi\left(a^{*} 1 a\right)=\phi\left(a_{k}^{*} \cdots a_{1}^{*} 1 a_{1} \cdots a_{k}\right) \\
& =\phi\left(a_{k}\right)^{*} \cdots \phi\left(a_{1}\right)^{*} \phi(1) \phi\left(a_{1}\right) \cdots \phi\left(a_{k}\right) \\
& =\left(\phi\left(a_{1}\right) \cdots \phi\left(a_{k}\right)\right)^{*} \phi(1)\left(\phi\left(a_{1}\right) \cdots \phi\left(a_{k}\right)\right) \\
& =b^{*} \phi(1) b \geq 0,
\end{aligned}
$$

where $b=\phi\left(a_{1}\right) \cdots \phi\left(a_{k}\right) \in B$. Thus, $\phi$ is positive linear and therefore a $*-$ homomorphism.

Next, we extend our nonexistence results to the nonunital case, by appealing to approximate unit arguments (which require continuity!) and the following important factorization property of $*$-preserving $n$-homomorphisms.

Lemma 4.3 (Coherent Factorization Lemma). Let $\phi: A \rightarrow B$ be an involutive n-homomorphism of $C^{\star}$-algebras. For any $1 \leq k \leq n$ and any $a \in A$, if $a=$ $a_{1} \cdots a_{k}=b_{1} \cdots b_{k}$ in $A$, then

$$
\phi\left(a_{1}\right) \cdots \phi\left(a_{k}\right)=\phi\left(b_{1}\right) \cdots \phi\left(b_{k}\right) \in B .
$$

Note that, in general, $\phi(a) \neq \phi\left(a_{1}\right) \cdots \phi\left(a_{k}\right)$ when $1<k<n$.

Proof. Clearly, we may assume $1<k<n$. Since $\phi$ is $*$-linear, the range $\phi(A) \subset B$ is a self-adjoint linear subspace of $B$ (but not necessarily a subalgebra, in general). Given any $d=\phi(c) \in \phi(A)$, using the Cohen Factorization Theorem, write $d=$ $d_{1} \cdots d_{n}=\phi\left(c_{1}\right) \cdots \phi\left(c_{n}\right)$ where $d_{i}=\phi\left(c_{i}\right)$ for $1 \leq i \leq n$. Consider the following computation:

$$
\begin{aligned}
\phi\left(a_{1}\right) \cdots \phi\left(a_{k}\right) d & =\phi\left(a_{1}\right) \cdots \phi\left(a_{k}\right) \phi\left(c_{1}\right) \cdots \phi\left(c_{n}\right) \\
& =\phi\left(a_{1} \cdots a_{k} c_{1} \cdots c_{n-k}\right) \phi\left(c_{n-k+1}\right) \cdots \phi\left(c_{n}\right) \\
& =\phi\left(b_{1} \cdots b_{k} c_{1} \cdots c_{n-k}\right) \phi\left(c_{n-k+1}\right) \cdots \phi\left(c_{n}\right) \\
& =\phi\left(b_{1}\right) \cdots \phi\left(b_{k}\right) \phi\left(c_{1}\right) \cdots \phi\left(c_{n}\right) \\
& =\phi\left(b_{1}\right) \cdots \phi\left(b_{k}\right) d .
\end{aligned}
$$

Let $f=\phi\left(a_{1}\right) \cdots \phi\left(a_{k}\right)-\phi\left(b_{1}\right) \cdots \phi\left(b_{k}\right)$. Then $f d=0$ for all $d \in \phi(A) \subset B$, and thus $f d=0$ for all $d$ in the $*$-subalgebra $A_{\phi}$ of $B$ generated by $\phi(A)$. In particular, for the element

$$
d_{a}=\phi\left(a_{k}^{*}\right) \cdots \phi\left(a_{1}^{*}\right)-\phi\left(b_{k}^{*}\right) \cdots \phi\left(b_{1}^{*}\right)=f^{*} \in A_{\phi} .
$$

Hence, $f f^{*}=f d_{a}=0$ and so $\|f\|^{2}=\left\|f f^{*}\right\|=0$ by the $C^{\star}$-identity. Therefore,

$$
\phi\left(a_{1}\right) \cdots \phi\left(a_{k}\right)-\phi\left(b_{1}\right) \cdots \phi\left(b_{k}\right)=f=0,
$$

and the result is proven. 
Definition 4.4. An approximate unit for a (nonunital) $C^{\star}$-algebra $A$ is a net $\left\{e_{\lambda}\right\}_{\lambda \in \Lambda}$ of elements in $A$ indexed by a directed set $\Lambda$ such that

(a) $0 \leq e_{\lambda}$ and $\left\|e_{\lambda}\right\| \leq 1$ for all $\lambda \in \Lambda$.

(b) $e_{\lambda} \leq e_{\mu}$ if $\lambda \leq \mu$ in $\Lambda$.

(c) For all $a \in A$,

$$
\lim _{\lambda \rightarrow \infty}\left\|a e_{\lambda}-a\right\|=\lim _{\lambda \rightarrow \infty}\left\|e_{\lambda} a-a\right\|=0 .
$$

Every $C^{\star}$-algebra has an approximate unit, which is countable $(\Lambda=\mathbb{N})$ if $A$ is separable (see Section II.4 of Blackadar [1]).

Theorem 4.5. Suppose $\phi: A \rightarrow B$ is an involutive $n$-homomorphism of $C^{\star}$ algebras, where $A$ is nonunital. Then, for all $a \in A$, the limit

$$
\psi(a)=\lim _{\lambda \rightarrow \infty} \phi\left(e_{\lambda}\right)^{n-2} \phi(a)=\lim _{\lambda \rightarrow \infty} \phi(a) \phi\left(e_{\lambda}\right)^{n-2}
$$

exists, independently of the choice of the approximate unit $\left\{e_{\lambda}\right\}$ of $A$, and defines $a *$-homomorphism $\psi: A \rightarrow B$ such that

$$
\phi(a)=\lim _{\lambda \rightarrow \infty} \phi\left(e_{\lambda}\right) \psi(a)
$$

for all $a \in A$.

Proof. We may assume $n \geq 3$. Given $a \in A$, use the Cohen Factorization Theorem to factor $a=a_{1} a_{2} \cdots a_{n}$. Define a map $\psi: A \rightarrow B$ by

$$
\psi(a)=\phi\left(a_{1} a_{2}\right) \phi\left(a_{3}\right) \cdots \phi\left(a_{n}\right)=\phi\left(a_{1}\right) \cdots \phi\left(a_{n-2}\right) \phi\left(a_{n-1} a_{n}\right),
$$

which is well-defined by the Coherent Factorization Lemma. The continuity of $\phi$ implies that

$$
\begin{aligned}
\lim _{\lambda \rightarrow \infty} \phi\left(e_{\lambda}\right)^{n-2} \phi(a) & =\lim _{\lambda \rightarrow \infty} \phi\left(e_{\lambda}\right)^{n-2} \phi\left(a_{1}\right) \cdots \phi\left(a_{n}\right) \\
& =\lim _{\lambda \rightarrow \infty} \phi\left(e_{\lambda}^{n-2} a_{1} a_{2}\right) \phi\left(a_{3}\right) \cdots \phi\left(a_{n}\right) \\
& =\phi\left(a_{1} a_{2}\right) \phi\left(a_{3}\right) \cdots \phi\left(a_{n}\right)=\psi(a) \in B .
\end{aligned}
$$

It follows that we can write:

$$
\psi(a)=\lim _{\lambda \rightarrow \infty} \phi\left(e_{\lambda}\right)^{n-2} \phi(a)=\lim _{\lambda \rightarrow \infty} \phi(a) \phi\left(e_{\lambda}\right)^{n-2},
$$

and so $\psi: A \rightarrow B$ is linear since $\phi$ is linear. Moreover, since $\phi$ is $*$-linear, it follows that $\psi$ is also $*$-linear:

$$
\begin{aligned}
\psi(a)^{*} & =\left(\phi\left(a_{1} a_{2}\right) \phi\left(a_{3}\right) \cdots \phi\left(a_{n}\right)\right)^{*} \\
& =\phi\left(a_{n}\right)^{*} \cdots \phi\left(a_{3}\right)^{*} \phi\left(a_{1} a_{2}\right)^{*} \\
& =\phi\left(a_{n}^{*}\right) \cdots \phi\left(a_{3}^{*}\right) \phi\left(a_{2}^{*} a_{1}^{*}\right) \\
& =\phi\left(a_{n 1}^{*} a_{n 2}^{*}\right) \phi\left(a_{n-1}^{*}\right) \cdots \phi\left(a_{12}^{*}\right) \\
& =\psi\left(\left(a_{n 1}^{*} a_{n 2}^{*}\right)\left(a_{n-1}^{*}\right) \cdots\left(a_{12}^{*}\right)\right) \\
& =\psi\left(a_{n}^{*} \cdots a_{1}^{*}\right)=\psi\left(a^{*}\right) .
\end{aligned}
$$

In the computation above, we factored $a_{n}=a_{n 2} a_{n 1}$ and set $a_{12}=a_{1} a_{2}$ to obtain the factorization $a^{*}=a_{n}^{*} \cdots a_{1}^{*}=\left(a_{n 1}^{*} a_{n 2}^{*}\right) a_{n-1}^{*} \cdots a_{12}^{*}$ into $n$ elements. Given 
$a, b \in A$ with factorizations $a=a_{1} \cdots a_{n}$ and $b=b_{1} \cdots b_{n}$, the fact that $\phi$ is an $n$-homomorphism implies:

$$
\begin{aligned}
\psi(a) \psi(b) & =\left(\phi\left(a_{1} a_{2}\right) \phi\left(a_{3}\right) \cdots \phi\left(a_{n}\right)\right)\left(\phi\left(b_{1} b_{2}\right) \phi\left(b_{3}\right) \cdots \phi\left(b_{n}\right)\right) \\
& =\phi\left(\left(a_{1} a_{2}\right) a_{3} \cdots a_{n}\left(b_{1} b_{2}\right)\right) \phi\left(b_{3}\right) \cdots \phi\left(b_{n}\right) \\
& =\phi\left(\left(a b_{1}\right) b_{2}\right) \phi\left(b_{3}\right) \cdots \phi\left(b_{n}\right) \\
& =\psi(a b)
\end{aligned}
$$

note that $a b=\left(a b_{1}\right) b_{2} b_{3} \cdots b_{n}$ is a factorization of $a b$ into $n$ elements. A second proof of multiplicativity goes as follows:

$$
\begin{aligned}
\psi(a b) & =\lim _{\lambda \rightarrow \infty} \phi\left(e_{\lambda}\right)^{n-2} \phi(a b)=\lim _{\lambda \rightarrow \infty} \phi\left(e_{\lambda}\right)^{n-2} \phi\left(\lim _{\mu \rightarrow \infty} a e_{\mu}^{n-2} b\right) \\
& =\lim _{\lambda \rightarrow \infty} \phi\left(e_{\lambda}\right)^{n-2} \lim _{\mu \rightarrow \infty} \phi\left(a e_{\mu}^{n-2} b\right) \\
& =\lim _{\lambda \rightarrow \infty} \phi\left(e_{\lambda}\right)^{n-2} \lim _{\mu \rightarrow \infty} \phi(a) \phi\left(e_{\mu}\right)^{n-2} \phi(b) \\
& =\lim _{\lambda \rightarrow \infty} \phi\left(e_{\lambda}\right)^{n-2} \phi(a) \lim _{\mu \rightarrow \infty} \phi\left(e_{\mu}\right)^{n-2} \phi(b) \\
& =\psi(a) \psi(b) .
\end{aligned}
$$

Thus, $\psi$ is a well-defined $*$-homomorphism. Finally, we compute:

$$
\begin{aligned}
\lim _{\lambda \rightarrow \infty} \phi\left(e_{\lambda}\right) \psi(a) & =\lim _{\lambda \rightarrow \infty} \phi\left(e_{\lambda}\right) \phi\left(a_{1} a_{2}\right) \phi\left(a_{3}\right) \cdots \phi\left(a_{n}\right) \\
& =\lim _{\lambda \rightarrow \infty} \phi\left(e_{\lambda}\left(a_{1} a_{2}\right) a_{3} \cdots a_{n}\right)=\lim _{\lambda \rightarrow \infty} \phi\left(e_{\lambda} a\right) \\
& =\phi(a) .
\end{aligned}
$$

Using similar factorizations, the fact that $\left\{e_{\lambda}^{n}\right\}$ is also an approximate unit for $A$, and the fact that the strict completion of the $C^{\star}$-algebra $C^{\star}(\phi(A))$ generated by the range $\phi(A)$ is the multiplier algebra $M\left(C^{\star}(\psi(A))\right)$, we obtain the nonunital version of Proposition A.1.

Corollary 4.6. Suppose that $A$ and $B$ are $C^{\star}$-algebras with $A$ nonunital, and let $\phi: A \rightarrow B$ be an involutive $n$-homomorphism with associated $*$-homomorphism $\psi: A \rightarrow B$. Then there is a self-adjoint n-potent $e=e^{*}=e^{n} \in M\left(C^{*}(\phi(A))\right)$ such that $\phi\left(e_{\lambda}\right) \rightarrow e$ strictly for any approximate unit $\left\{e_{\lambda}\right\}$ of $A$, and with the property that

for all $a \in A$.

$$
\begin{aligned}
& \phi(a)=e \psi(a)=\psi(a) e, \\
& \psi(a)=e^{n-2} \phi(a)
\end{aligned}
$$

Proof. By the previous proof, we can define $e \in M\left(C^{\star}(\phi(A))\right)$ on generators $\phi(a)$ by

$$
e \phi(a)=\lim _{\lambda \rightarrow \infty} \phi\left(e_{\lambda}\right) \phi(a)=\phi\left(a_{1} a_{2} \cdots a_{n-1}\right) \phi\left(a_{n}\right) \in C^{\star}(\phi(A))
$$

for any $a=a_{1} \cdots a_{n} \in A$. It follows that:

$$
\begin{aligned}
e^{n} \phi(a) & =\lim _{\lambda \rightarrow \infty} \phi\left(e_{\lambda}\right)^{n} \phi(a) \\
& =\lim _{\lambda \rightarrow \infty} \phi\left(e_{\lambda}^{n}\right) \phi\left(a_{1}\right) \phi\left(a_{2}\right) \cdots \phi\left(a_{n}\right) \\
& =\lim _{\lambda \rightarrow \infty} \phi\left(\left(e_{\lambda}^{n}\right) a_{1} a_{2} \cdots a_{n-1}\right) \phi\left(a_{n}\right) \\
& =\phi\left(a_{1} \cdots a_{n-1}\right) \phi\left(a_{n}\right)=e \phi(a),
\end{aligned}
$$


which implies $e \in M\left(C^{\star}(\phi(A))\right)$ is $n$-potent. The fact that $e=e^{*}$ follows from $\phi\left(e_{\lambda}\right)^{*}=\phi\left(e_{\lambda}^{*}\right)=\phi\left(e_{\lambda}\right)$. The other statements follow from the previous proof.

The dichotomy between the unital and nonunital cases is now clear. If $A$ is unital, then $C^{\star}(\phi(A)) \subset B$ is a unital $C^{\star}$-subalgebra of $B$ with unit $\psi(1)=\phi(1)^{n-1} \in B$ (which is a projection!) and so

$$
M\left(C^{\star}(\psi(A))\right)=C^{\star}(\phi(A)) \subset B .
$$

However, for $A$ nonunital, we cannot identify the multiplier algebra $M\left(C^{\star}(\phi(A))\right)$ as a subalgebra of $B$, or even $M(B)$, unless $\phi$ is surjective. In general, we only have inclusions $\psi(A) \subset C^{\star}(\phi(A)) \subset B$.

Now that we know, as in the unital case, every involutive $n$-homomorphism is an $n$-potent multiple of a $*$-homomorphism, we can prove the following general version of Theorem 4.1 and its corollary in a similar manner using Lemma A.8.

Theorem 4.7. Let $\phi: A \rightarrow B$ be an involutive $n$-homomorphism of $C^{\star}$-algebras. If $n \geq 2$ is even, then $\phi$ is $a *$-homomorphism. If $n \geq 3$ is odd, then $\phi$ is the difference $\phi(a)=\psi_{1}(a)-\psi_{2}(a)$ of two orthogonal $*$-homomorphisms $\psi_{1} \perp \psi_{2}: A \rightarrow B$.

Corollary 4.8. For all $n \geq 2$ and $C^{\star}$-algebras $A$ and $B, \phi: A \rightarrow B$ is a positive $n$-homomorphism if and only if $\phi$ is a $*$-homomorphism.

\section{ApPENDiX A. ON $n$-HOMOMORPHISMS AND $n$-POTENTS}

An element $x \in A$ is called an $n$-potent if $x^{n}=x$. Note that if $\phi: A \rightarrow B$ is an $n$ homomorphism, then $\phi(x)=\phi\left(x^{n}\right)=\phi(x)^{n} \in B$ is also an $n$-potent. The following important result is Proposition 2.2 in [7, whose proof is included for completeness.

Proposition A.1. If $A$ is a unital algebra (or ring) and $\phi: A \rightarrow B$ is an nhomomorphism, then there is a homomorphism $\psi: A \rightarrow B$ and an n-potent $e=$ $e^{n} \in B$ such that $\phi(a)=e \psi(a)=\psi(a) e$ for all $a \in A$. Also, $e$ commutes with the rang 1 of $\phi$, i.e., $e \phi(a)=\phi(a) e$ for all $a \in A$.

Proof. Note that $e=\phi(1)=\phi\left(1^{n}\right)=\phi(1)^{n}=e^{n} \in B$ is an $n$-potent. Define a linear map $\psi: A \rightarrow B$ by $\psi(a)=e^{n-1} \phi(a)$ for all $a \in A$. For all $a, b \in A$,

$$
\begin{aligned}
\psi(a b) & =e^{n-2} \phi(a b)=e^{n-2} \phi\left(a 1^{n-2} b\right) \\
& =\left(e^{n-2} \phi(a)\right)\left(\phi(1)^{n-2} \phi(b)\right) \\
& =\left(e^{n-2} \phi(a)\right)\left(e^{n-2} \phi(b)\right)=\psi(a) \psi(b),
\end{aligned}
$$

and so $\psi$ is an algebra homomorphism. Furthermore,

$$
e \psi(a)=\phi(1)\left(\phi(1)^{n-2} \phi(a)\right)=\phi(1)^{n-1} \phi(a)=\phi\left(1^{n-1} a\right)=\phi(a) .
$$

Similarly, $\psi(a) e=\phi(a)$ for all $a \in A$. The final statement is a consequence of the fact that for all $a \in A$,

$$
e \phi(a)=\phi(1) \phi\left(a 1^{n-1}\right)=\left(\phi(1) \phi(a) \phi(1)^{n-2}\right) \phi(1)=\phi\left(1 a 1^{n-2}\right) e=\phi(a) e .
$$

The following computation will be more significant when we consider the nonunital case (see the proof of Theorem 4.5).

\footnotetext{
${ }^{1}$ Note that the range $\phi(A)$ is not a subalgebra of $B$ in general.
} 
Corollary A.2. Let $\phi$ and $\psi$ be as in Proposition A.1 and $n \geq 3$. Then for all $a \in A$, if $a=a_{1} a_{2} \cdots a_{n}$ with $a_{1}, \ldots, a_{n} \in A$,

$$
\psi(a)=\phi\left(a_{1} a_{2}\right) \phi\left(a_{3}\right) \cdots \phi\left(a_{n}\right) .
$$

Proof. We compute as follows:

$$
\begin{aligned}
\psi(a) & =\operatorname{def}^{n-2} \phi(a)=\phi(1)^{n-2} \phi\left(a_{1} \cdots a_{n}\right) \\
& =\phi(1)^{n-2} \phi\left(a_{1}\right) \cdots \phi\left(a_{n}\right) \\
& =\left(\phi(1)^{n-2} \phi\left(a_{1}\right) \phi\left(a_{2}\right)\right) \phi\left(a_{3}\right) \cdots \phi\left(a_{n}\right) \\
& =\phi\left(1^{n-2} a_{1} a_{2}\right) \phi\left(a_{3}\right) \cdots \phi\left(a_{n}\right) \\
& =\phi\left(a_{1} a_{2}\right) \phi\left(a_{3}\right) \cdots \phi\left(a_{n}\right) .
\end{aligned}
$$

Definition A.3. Let $A$ be a unital algebra. An $n$-partition of unity is an ordered $n$-tuple $\left(e_{0}, e_{1}, \ldots, e_{n-1}\right)$ of idempotents $\left(e_{k}^{2}=e_{k}\right)$ that sum to the identity $e_{0}+$ $e_{1}+\cdots+e_{n-1}=1$ and are pairwise mutually orthogonal, i.e., $e_{j} e_{k}=\delta_{j k} 1$ for all $0 \leq j, k \leq n-1$, where $\delta_{j k}$ is the Kronecker delta.

Note that $e_{0}=1-\left(e_{1}+\cdots+e_{n-1}\right)$ is completely determined by $e_{1}, e_{2}, \ldots, e_{n-1}$ and is thus redundant in the notation for an $n$-partition of unity.

Definition A.4. Let $\omega_{0}=0$ and $\omega_{k}=e^{2 \pi i(k-1) /(n-1)}$ for $1 \leq k \leq n-1$. Note that $\omega_{1}=1$ and $\omega_{1}, \ldots, \omega_{n-1}$ are the $(n-1)$-th roots of unity and $\Sigma_{n}=$ $\left\{\omega_{0}, \omega_{1}, \ldots, \omega_{n-1}\right\}$ are the $n$ roots of the polynomial equation $x^{n}-x=x\left(x^{n-1}-1\right)=$ 0 .

If $A$ is a complex algebra, we let $\tilde{A}$ denote $A$, if $A$ is unital, or the unitization $A^{+}=A \oplus \mathbb{C}$, if $A$ is nonunital.

Theorem A.5. Let $A$ be a complex algebra. If $e \in A$ is an n-potent, there is a unique $n$-partition of unity $\left(e_{0}, e_{1}, \ldots, e_{n-1}\right)$ in $\tilde{A}$ such that

$$
e=\sum_{k=1}^{n-1} \omega_{k} e_{k} .
$$

If $A$ is nonunital, then $e_{1}, \ldots, e_{n-1} \in A$.

Proof. Define the $n$ polynomials $p_{0}, p_{1}, \ldots, p_{n-1}$ by

$$
p_{k}(x)=\frac{\prod_{j \neq k}\left(x-\omega_{j}\right)}{\prod_{j \neq k}\left(\omega_{k}-\omega_{j}\right)} .
$$

In particular, $p_{0}(x)=1-x^{n-1}$. Each polynomial $p_{k}$ has degree $n-1$ and satisfies $p_{k}\left(\omega_{k}\right)=1$ and $p_{k}\left(\omega_{j}\right)=0$ for all $j \neq k$. It follows that $p_{j}(x) p_{k}(x)=0$ for all $x \in \Sigma_{n}$. We also claim for all $x \in \mathbb{C}$ that

$$
\begin{aligned}
\sum_{k=0}^{n-1} p_{k}(x) & =p_{0}(x)+\cdots+p_{n-1}(x)=1 . \\
x & =\sum_{k=0}^{n-1} \omega_{k} p_{k}(x) .
\end{aligned}
$$

Indeed, these identities follow from the fact that these polynomial equations have degree $n-1$ but are satisfied by the $n$ distinct points in $\Sigma_{n}$. 
Now, given any $x^{n}=x$ in $\mathbb{C}$ it follows that $p_{k}(x)^{2}=p_{k}(x)$. Hence, for any $n$ potent $e \in A$, if we define $e_{k}=p_{k}(e)$, then $\left(e_{0}, e_{1}, \ldots, e_{n-1}\right)$ consists of idempotents $e_{k}^{2}=p_{k}(e)^{2}=p_{k}(e)=e_{k}$ and satisfy, by (2),

$$
\sum_{k=0}^{n-1} e_{k}=\sum_{k=0}^{n-1} p_{k}(e)=1_{\tilde{A}}
$$

They are pairwise orthogonal because $e_{j} e_{k}=p_{j}(e) p_{k}(e)=0$ for $j \neq k$. Moreover,

$$
e=\sum_{k=1}^{n-1} \omega_{k} p_{k}(e)=\sum_{k=1}^{n-1} \omega_{k} e_{k}
$$

by Equation (3). For $1 \leq k \leq n-1$, note that $p_{k}(x)=x q_{k}(x)$ for some polynomial $q_{k}(x)$. Hence, if $A$ is nonunital and $1 \leq k \leq n-1$, we have $e_{k}=p_{k}(e)=e q_{k}(e) \in A$, since $A$ is an ideal in $\tilde{A}$.

The following result is the $n$-homomorphism version of the previous $n$-potent result. Recall say that two linear maps $\psi_{i}, \psi_{j}: A \rightarrow B$ are orthogonal $\left(\psi_{i} \perp \psi_{j}\right)$ if

$$
\psi_{i}(a) \psi_{j}(b)=\psi_{j}(b) \psi_{i}(a)=0
$$

for all $a, b \in A$.

Proposition A.6. Let $A$ and $B$ be complex algebras. If $A$ is unital, then a linear map $\phi: A \rightarrow B$ is an $n$-homomorphism if and only if there exist $n-1$ mutually orthogonal homomorphisms $\psi_{1}, \ldots, \psi_{n-1}: A \rightarrow B$ such that for all $a \in A$,

$$
\phi(a)=\sum_{k=1}^{n-1} \omega_{k} \psi_{k}(a) .
$$

Proof. $(\Rightarrow)$ Let $\phi: A \rightarrow B$ be an $n$-homomorphism. By Proposition A.1, there is an $n$-potent $e \in B$ and a homomorphism $\psi: A \rightarrow B$ such that $\phi(a)=e \psi(a)=\psi(a) e$. Using the previous result, write $e=\sum_{k=1}^{n-1} \omega_{k} e_{k}$, where $\left(e_{0}, e_{1}, \ldots, e_{n-1}\right)$ is the associated $n$-partition of unity in $\tilde{A}$ defined by the polynomials $p_{k}$. Since $e_{k}=p_{k}(e)$, we have that $e_{k} \psi(a)=\psi(a) e_{k}$ for $1 \leq k \leq n-1$. Define $\psi_{k}: A \rightarrow B$ by

$$
\psi_{k}(a)={ }_{\operatorname{def}} e_{k} \psi(a)=e_{k}^{2} \psi(a)=e_{k} \psi(a) e_{k} .
$$

Then $\psi_{1}, \ldots, \psi_{n-1}$ are orthogonal homomorphisms and, for all $a \in A$,

$$
\phi(a)=e \psi(a)=\sum_{k=1}^{n-1} \omega_{k} e_{k} \psi(a)=\sum_{k=1}^{n-1} \omega_{k} \psi_{k}(a) .
$$

$(\Leftarrow)$ Follows from the fact that $\omega_{k}^{n}=\omega_{k}$ for all $k=1, \ldots, n-1$.

Remark A.7. If $A$ is nonunital, the above result does not hold. One reason is that the unitization $\phi^{+}: A^{+} \rightarrow B^{+}$of an $n$-homomorphism is not, in general, an $n$ homomorphism. Also, if $A^{n}=B^{n}=\{0\}$, then every linear map $L: A \rightarrow B$ is an $n$-homomorphism. (See Examples 2.5 and 4.3 of Hejazian et. al. [7.)

Let $\Sigma_{n}$ be the $n$ roots of the polynomial equation $x=x^{n}$ from Definition A.4. If $A$ is a $C^{\star}$-algebra, it follows that a normal $n$-potent $e=e^{n}$ must have spectrum $\sigma(e) \subseteq \Sigma_{n}$. Recall that a projection is an element $p=p^{*}=p^{2} \in A$. Two projections $p_{1}$ and $p_{2}$ are orthogonal if $p_{1} p_{2}=0$. A tripotent is a 3 -potent element $e^{3}=e \in A$.

${ }^{2}$ Note that the zero homomorphism is orthogonal to every homomorphism. 
The following characterization of self-adjoint $n$-potents in $C^{\star}$-algebras is important for our nonexistence results on $n$-homomorphisms.

Lemma A.8. Let $A$ be a $C^{\star}$-algebra.

(a) If $n \geq 2$ is an even integer, the following are equivalent:

(i) $e$ is a projection.

(ii) $e$ is a positive n-potent.

(iii) $e$ is a self-adjoint n-potent.

(b) If $n \geq 3$ is an odd integer, the following are equivalent:

(i) $e$ is a self-adjoint tripotent.

(ii) $e=p_{1}-p_{2}$ is a difference of two orthogonal projections.

(iii) $e$ is a self-adjoint n-potent.

Proof. In both the even and odd cases, (i) $\Longrightarrow$ (ii) $\Longrightarrow$ (iii) (See Theorem A.5.) Suppose (iii) holds. If $n=2 k$ is even,

$$
e=e^{*}=e^{n}=e^{2 k}=\left(e^{k}\right)^{*}\left(e^{k}\right) \geq 0,
$$

and so the spectrum of $e$ satisfies $\sigma(e) \subset \Sigma_{n} \cap[0, \infty]=\{0,1\}$. Thus, $e$ is a projection. If $n \geq 3$ is odd, then since $e=e^{*}$ we must have $\sigma(e) \subset \Sigma_{n} \cap \mathbb{R}=\{-1,0,1\}$. Thus, $\lambda=\lambda^{3}$ for all $\lambda \in \sigma(e)$, which implies $e=e^{3}$ is tripotent.

\section{REFERENCES}

[1] B. Blackadar, Theory of $C^{*}$-algebras and von Neumann algebras, Encyclopaedia of Mathematical Sciences, 122. Operator Algebras and Non-commutative Geometry, III. SpringerVerlag, Berlin, 2006. MR2188261 (2006k:46082)

[2] J. Bračič and S. Moslehian, On Automatic Continuity of 3-Homomorphisms on Banach Algebras, to appear in Bull. Malays. Math. Sci. Soc. arXiv: math.FA/0611287.

[3] P. Cohen, Factorization in group algebras, Duke Math. J. 26 (1959) 199-205. MR0104982 $(21: 3729)$

[4] S. Feigelstock, Rings whose additive endomorphisms are N-multiplicative, Bull. Austral. Math. Soc. 39 (1989), no. 1, 11-14. MR976254 (89j:16043)

[5] S. Feigelstock, Rings whose additive endomorphisms are $N$-multiplicative. II, Period. Math. Hungar. 25 (1992), no. 1, 21-26. MR1200838 (93j:16018)

[6] L. Harris, A Generalization of $C^{\star}$-algebras, Proc. London Math. Soc. 42 (1981), no. 3, 331361. MR607306 (82e:46089)

[7] M. Hejazian, M. Mirzavaziri, and M.S. Moslehian, n-homomorphisms, Bull. Iranian Math. Soc. 31 (2005), no. 1, 13-23. MR2228453 (2007b:47091)

[8] I. Raeburn and D. P. Williams, Morita Equivalence and Continuous-Trace $C^{\star}$-Algebras, Mathematical Surveys and Monographs, vol. 60, American Mathematical Society, 1998. MR:1634408 (2000c:46108)

[9] W. Stinespring, Positive functions on $C^{\star}$-algebras, Proc. Amer. Math. Soc. 6 (1955), 211-216. MR0069403 (16:1033b)

Department of Mathematics, Texas Christian University, Box 298900, Fort Worth, TEXAs 76129

E-mail address: e.park@tcu.edu

Department of Mathematics, Dartmouth College, 6188 Kemeny Hall, Hanover, New HAMPSHIRE 03755

E-mail address: jody.trout@dartmouth.edu 